\title{
Restricted homogeneity implies bi-additivity
}

\author{
by T. M. K. Davison (Hamilton, Ontario, Canada)
}

Abstract. Let $R$ be a commutative ring with identity, and let $M$ be an $R$-module. Suppose $F: M \times M \rightarrow R$ satisfies

$$
F(x, y)+F(x+y, z)=F(x, y+z)+F(y, z)
$$

and

$$
F(0,0)=0
$$

Two results are proved.

THEOREM 1. Let $r, s$ be fixed elements of $R$ such that $r s$ is not a zero divisor, and $r-s$ is an invertible element of $R$. If $F$ satisfies (1), (2) and

$$
F(r x, s y)=r s F(x, y),
$$

then $F$ is additive in each variable.

THEOREM 2. Let $\boldsymbol{R}$ be the ring $\boldsymbol{Z}$ of rational integers. Let $r, s$ be distinct non-zero integers. If $F$ satisfies (1), (2) and (3), then $F$ is bi-additive.

Theorem 2 yields the result due to Jordan and von Neumann, that if $f: M \rightarrow Z$ satisfies the parallelogram law, then $F(x, y)=f(x+y)-f(x)-f(y)$ is bi-additive.

A commonly accepted definition of quadratic forms is the following (see e.g. Jacobson [1], Definition 6.1), where $R$ is a commutative ring with identity, and $M$ is a (unitary) $R$-module. A function $f: M \rightarrow R$ is a quadratic form if

(i) $f(r x)=r^{2} f(x)$ for all $r \in R, x \in M$,

(ii) $F: M \times M \rightarrow R$ is bilinear, where, for all $x, y \in M$,

$$
F(x, y):=f(x+y)-f(x)-f(y) \text {. }
$$

The second requirement can be separated into two parts: that $F$ be homogeneous (of degree one) in each variable, and that $F$ be bi-additive. It will be shown that suitable homogeneity of $F$, and the fact (a consequence of its definition by (1)) that $F$ satisfies

$$
F(x, y)+F(x+y, z)=F(x, y+z)+F(y, z)
$$

implies that $F$ is bi-additive. 
The particular situation which motivates and illuminates our treatment is the deduction of bi-additivity from the parallelogram law (cf. Jordan-von Neumann [2], Theorem 1). Suppose $f: M \rightarrow R$ satisfies

$$
f(x+y)+f(x-y)=2 f(x)+2 f(y)
$$

and 2 is not a zero divisor in $R$, then $F$ satisfied the partial homogeneity condition

$$
F(x,-y)=-F(x, y) .
$$

Bi-additivity of $F$ is proved in

Proposition 1. Let $R$ be a ring in which 2 is not a zero divisor. Let $M$ be an $R$-module and suppose $F: M \times M \rightarrow R$ satisfies (2) and (4). Then $F$ is biadditive.

Proof. Substitute $-z$ for $z$ in (2) to obtain

$$
F(x, y)+F(x+y,-z)=F(x, y-z)+F(y,-z) .
$$

Add (2) and (5), and use (4) to deduce that

$$
2 F(x, y)=F(x, y+z)+F(x, y-z) .
$$

In (6) interchange $y$, and $z$, and add the resulting equation to (6) to deduce (using (4) again) that

$$
2 F(x, y)+2 F(x, z)=2 F(x, y+z) .
$$

Since 2 is not a zero divisor, (7) yields the additivity of $F$ in the second variable. A further use of (2) yields the additivity of $F$ in the first variable. Hence $F$ is bi-additive.

This result is generalized in

Proposition 2. Let $r, s$ be fixed elements of $R$ such that $r s$ is not a zero divisor. If $F: M \times M \rightarrow R$ satisfies (2)

$$
F(r x, s y)=r s F(x, y)
$$

and

$$
F(0,0)=0
$$

then

$$
F(x+t y, z)=F(x, z)+F(t y, z)
$$

for all $x, y, z \in M$, where $t:=r-s$.

Remark. If $r s-1$ is not a zero divisor, then (9) is a consequence of (8). If $F$ satisfies (2) and (8), then $F-F(0,0)$ satisfies (2), (8) and (9). 
Proof. In (2) replace $x$ by $r x, y$ by $r s y$, and $z$ by $s z$ to obtain

$$
F(r x, r s y)+F(r x+r s y, s z)=F(r x, r s y+s z)+F(r s y, s z) .
$$

Simplify (11) using (8) and cancel the $r s$ factors resulting to deduce that

$$
F(x, r y)+F(x+s y, z)=F(x, r y+z)+F(s y, z) .
$$

However, writing $r y$ for $y$ i (2) yields

$$
F(x, r y)+F(x+r y, z)=F(x, r y+z)+F(r y, z) .
$$

Subtracting (12) from (13), and using the notation $t:=r-s$ we have

$$
F(x+s y+t y, z)-F(x+s y, z)=F(s y+t y, z)-f(s y, z) .
$$

Set $x=-s y$ in (14), and use the fact that $F\left(x^{\prime}, 0\right)=F\left(0, z^{\prime}\right)=0$ by (9) and (2), to obtain $F(s y+t y, z)-F(s y, z)=F(t y, z)$; so (14) can be rewritten

$$
F(x+s y+t y, z)-F(x+s y, z)=F(t y, z) .
$$

Finally in (15) replace $x+s y$ by $x$ to deduce (10).

Corollary. Suppose $F$ satisfies (2), (8), and (9); and $r-s$ is an invertible element of $R$, then $F$ is bi-additive. In particular, if $R$ is a field with at least 3 elements and $F$ satisfies (2), (8) and (9) for a pair $r \neq 0, s \neq 0, r \neq s$, then $F$ is bi-additive.

Proof. Write $t^{-1} y$ for $y$ in (10).

If one considers the corollary applied to $r=1, s=-1$ one sees that one has to assume that 2 is invertible to deduce the additivity of $F$, whereas in Proposition 1 all one requires in that 2 not be a zero divisor. It seems that the fact that $r$ and $s$ are in the subring of $R$ generated by 1 is critical, as is shown in the next (and final) result.

Proposition 3. Let $r, s$ be fixed elements of $Z$ (the ring of rational integers) such that $r s \neq 0$, and $r \neq s$. If $F: M \times M \rightarrow Z$ satisfies (2), (8) and (9), then $F$ is bi-additive.

Proof. We can assume that $|r s|>1$, as $r s=1$ violates the assumption $r \neq s$, and $r s=-1$ is taken care of in Proposition 1.

We exploit (10) by writing $(t:=r-s$ as usual),

$$
r s F(x, y)=F(r x, s y)=F(s x+t x, s y)=F(s x, s y)+F(t x, s y) .
$$

Now by (2)

$$
F(t x, y)+F(t x+y, z)=F(t x, y+z)+F(y, z)
$$

so using (10) here again

$$
F(t x, y)+F(t x, z)=F(t x, y+z) .
$$


Hence by (17), and the fact that $s \in Z$

$$
F(t x, s y)=s F(t x, y) \text {. }
$$

Indeed, for all $m, n \in Z$,

$$
F(m t x, n y)=m n F(t x, y) .
$$

The outcome of all this is that (16) may be rewritten as

$$
F(s x, s y)=r s F(x, y)-s F(t x, y) .
$$

For each $n \geqslant 0$ define $\alpha_{n}, \beta_{n} \in Z$ by $\alpha_{0}=1, \beta_{0}=0$ and

$$
\alpha_{n+1}=r s \cdot \alpha_{n}, \quad \beta_{n+1}=r s \cdot \beta_{n}+s^{2 n+1}
$$

for $n \geqslant 1$. Then it is an easy exercise to use (20) and (21) to prove by induction, that for all $n \geqslant 0$

$$
F\left(s^{n} x, s^{n} y\right)=\alpha_{n} F(x, y)-\beta_{n} F(t x, y) .
$$

It is also easy to prove from (21) that

$$
\alpha_{n}-t \beta_{n}=s^{2} n \text {. }
$$

Since $t \neq 0$, there are by Euler's theorem (or the fact that $Z / t Z$ is a finite ring), positive integers $m>n$ such that $s^{m} \equiv s^{n} \bmod t$ say $s^{m}=s^{n}+u t$. Then on the one hand by (22)

$$
F\left(s^{m} x, s^{m} y\right)=\alpha_{m} F(x, y)-\beta_{m} F(t x, y)
$$

and on the other hand, using (10),

$$
\begin{aligned}
F\left(s^{m} x, s^{m} y\right) & =F\left(s^{n} x+u t x, s^{n} y+u t y\right) \\
& =F\left(s^{n} x, s^{n} y\right)+F\left(s^{n} x, u t y\right)+F\left(u t x, s^{n} y\right)+F(u t x, u t y) \\
& =\alpha_{n} F(x, y)-\beta_{n} F(t x, y)+u s^{n} F(x, t y)+ \\
& \quad+u s^{n} F(t x, y)+u^{2} t F(t x, y)
\end{aligned}
$$

by repeated use of (17). Moreover, $F(t x, t y)=t F(t x, y)$ and $F(t x, t y)$ $=t F(x, t y)$, by (19). Since $t \neq 0$, we see that $F(t x, y)=F(x, t y)$. Thus our second evaluation of $F\left(s^{m} x, s^{m} y\right)$ is $\alpha_{n} F(x, y)-\left(\beta_{n}-2 u s^{n}-u^{2} t\right)=F(t x, y)$. Equating the two evaluations, and rearranging, we obtain

$$
\left(\beta_{m}-\beta_{n}+2 u s^{n}+u^{2} t\right) F(t x, y)=\left(\alpha_{m}-\alpha_{n}\right) F(x, y) .
$$

Multiply both sides of this by $t$, and use (23) to deduce.

$$
\left(\alpha_{m}-\alpha_{n}\right) F(t x, y)=\left(\alpha_{m}-\alpha_{n}\right) t F(x, y) .
$$


Finally, $\alpha_{m}-\alpha_{n}=(r s)^{m}-(r s)^{n} \neq 0$ since $\{r s\}>1$ and $m>n$. So we deduce from (25) that

$$
F(t x, y)=t F(x, y)
$$

Now use (17) and (26) to infer that

$$
t F(x, y+z)=t F(x, y)+t F(x, z) ;
$$

cancelling $t$ (since $t$ is not a zero divisor) yields the desired result.

\section{References}

[1] N. Jacobson, Basic Algebra l, W. H. Freeman, San Francisco 1974.

[2] P. Jordan, and J. von Neumann, On inner products in linear metric space, Ann. of Math. 36 (1935), 719-723.

DEPARTMENT OF MATHEMATICAL SCIENCES

MCMASTER UNIVERSITY, HAMILTON, ONTARIO, CANADA

Reçu par la Rédaction le 1979.07.31 